\title{
Intraoperative somatosensory evoked potential recovery following opening of the fourth ventricle during posterior fossa decompression in Chiari malformation: case report
}

\author{
Stefan Grossauer, MD, Katharina Koeck, MD, and Giles H. Vince, MD \\ Department of Neurosurgery, General Hospital of Klagenfurt, Austria
}

The most appropriate surgical technique for posterior fossa decompression in Chiari malformation (CM) remains a matter of debate. Intraoperative electrophysiological studies during posterior fossa decompression of Type I CM (CM-I) aim to shed light on the entity's pathomechanism as well as on the ideal extent of decompression. The existing reports on this issue state that significant improvement in conduction occurs after craniotomy in all cases, but additional durotomy contributes a further improvement in only a minority of cases. This implies that craniotomy alone might suffice for clinical improvement without the need of duraplasty or even subarachnoid manipulation at the level of the craniocervical junction.

In contrast to published data, the authors describe the case of a 32-year-old woman who underwent surgery for CM associated with extensive cervicothoracic syringomyelia and whose intraoperative somatosensory evoked potentials (SSEPs) did not notably improve after craniotomy or following durotomy; rather, they only improved after opening of the fourth ventricle and restoration of CSF flow through the foramen of Magendie. Postoperatively, the patient recovered completely from her preoperative neurological deficits. To the authors' knowledge, this is the first report of significant SSEP recovery after opening the fourth ventricle in the decompression of a CM-I. The electrophysiological and operative techniques are described in detail and the findings are discussed in the light of available literature. The authors conclude that there might be a subset of $\mathrm{CM}-\mathrm{I}$ patients who require subarachnoid dissection at the level of the craniocervical junction to benefit clinically. Prospective studies with detailed electrophysiological analyses seem warranted to answer the question regarding the best surgical approach in CM-I decompression.

http://thejns.org/doi/abs/10.3171/2014.10.JNS14401

KEY WORDS somatosensory evoked potentials; posterior fossa; Chiari malformation; duraplasty; intraoperative neuromonitoring

$\mathrm{T}$ HE most appropriate surgical technique for posterior fossa decompression in Chiari malformation (CM) remains controversial. Although the majority of neurosurgeons perform a bony decompression with duraplasty for symptomatic patients, there may be a subset of patients who improve in response to bony decompression alone, and others may even require a more extensive manipulation at the cerebellar tonsils and fourth ventricle outlet. ${ }^{4,7,9}$ Intraoperative electrophysiological monitoring is not only applied in Chiari Type I malformation (CM-I) decompression to prevent neurological deficit but is also employed to shed light on the pathomechanism of the entity and the ideal extent of CM-I decompression. The exist- ing reports on this issue state that significant improvement in conduction occurs in all cases after craniotomy and removal of the dural band alone, and only a few patients benefit from additional durotomy. ${ }^{1,2,5,14}$ The authors conclude that these intraoperative electrophysiological data indicate the most significant surgical step that is associated with clinical improvement - that is, bony decompression and to a lesser extent durotomy or duraplasty.

In contrast to published data, we describe the case of an adult patient who underwent surgery for CM with extensive cervicothoracic syringomyelia and whose intraoperative somatosensory evoked potentials (SSEPs) did not improve significantly after craniotomy or following du-

ABBREVIATIONS BAEP = brainstem auditory evoked potential; $\mathrm{CM}=$ Chiari malformation; $\mathrm{CM}-\mathrm{I}=\mathrm{CM}$ Type I; SSEP = somatosensory evoked potential. SUBMITTED February 20, 2014. ACCEPTED October 30, 2014.

INCLUDE WHEN CITING Published online December 19, 2014; DOI: 10.3171/2014.10.JNS14401.

DISCLOSURE The authors report no conflict of interest concerning the materials or methods used in this study or the finding specified in this paper. 
rotomy, as described in the literature, but only improved after we opened the fourth ventricle and established CSF flow through the foramen of Magendie.

\section{Case Report}

Presentation

A 32-year-old woman with a 3-year history of occipital headache, numbness in both hands, and gait disorder consulted our department. Neurological examination revealed dissociated sensory disturbance in the upper extremities and trunk, as well as severe spinal ataxia. Magnetic resonance imaging revealed the typical features of CM associated with extensive cervicothoracic syringomyelia (Fig. 1). Because of these findings, associated with progressive neurological deterioration, a posterior fossa decompression was indicated.

\section{Operation}

The patient was positioned prone with her head slightly flexed after induction of general anesthesia. After making a midline skin incision from inion to $\mathrm{C}-2$, we dissected the paraspinal muscles from the bone and harvested pericranium for later duraplasty. A small median suboccipital craniotomy extending only $3.5 \mathrm{~cm}$ cranially and $2 \mathrm{~cm}$ to both sides laterally, including the foramen magnum caudally, and a C-1 laminectomy were performed. The dura was opened in a $\mathrm{Y}$ shape and the underlying cerebellar tonsils were separated in the midline by dissecting the adherent arachnoid. Abnormal arachnoid adhesions between the cerebellar tonsils and the medulla oblongata were encountered and were dissected to establish CSF flow. After shrinking the tonsils by applying bipolar cautery, the obex and the lower part of the fourth ventricle could be visualized through the foramen of Magendie (Fig. 2). Cerebrospinal fluid flow was established at the level of the craniocervical junction and a duraplasty with a pericranial graft was performed in watertight fashion with 4-0 nylon
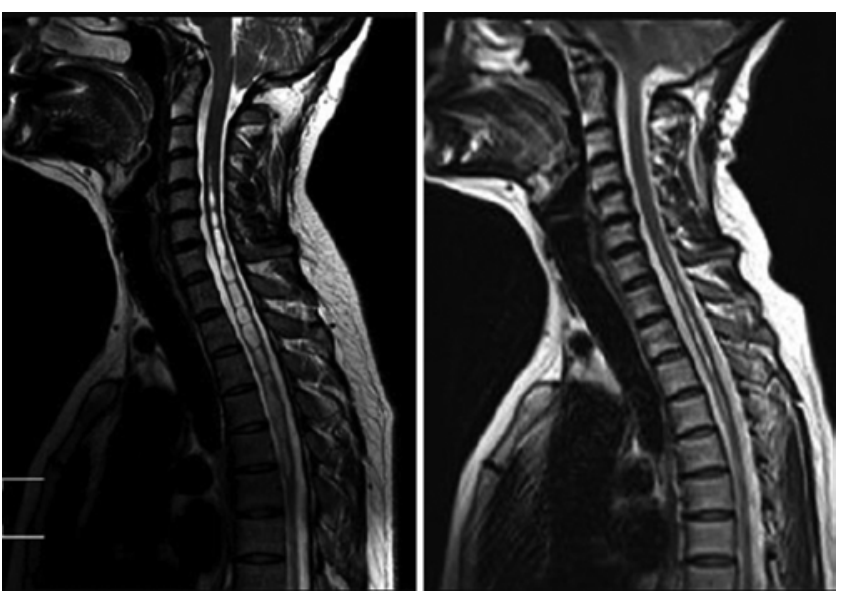

FIG. 1. Sagittal T2-weighted MR images. Left: Preoperative image illustrating the typical features of the $\mathrm{CM}$ with tonsillar herniation below the foramen magnum and associated extensive cervicothoracic syringomyelia. Right: Postoperative image demonstrating reestablished CSF flow at the craniocervical junction and significant reduction in syringomyelia a few weeks after the operation.

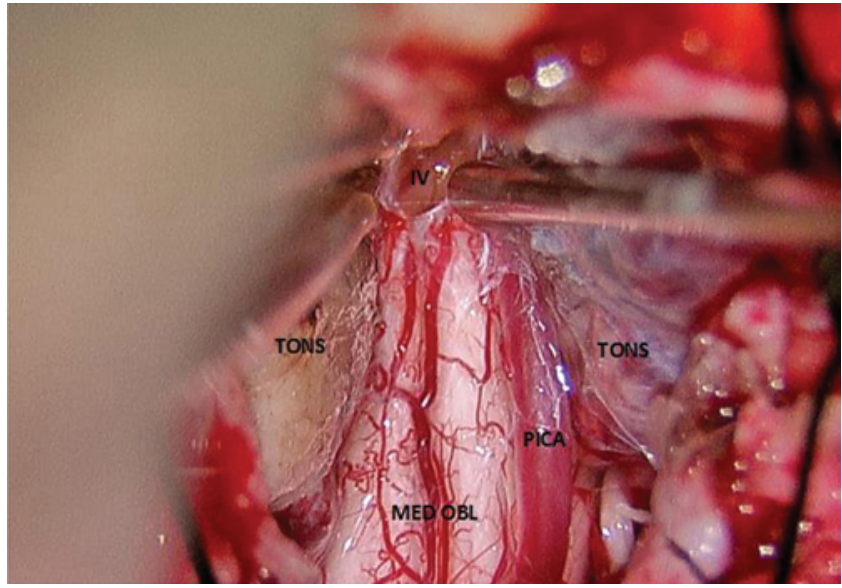

FIG. 2. Intraoperative microscopic view into the lower portion of the fourth ventricle through the foramen of Magendie after reduction in the size of the cerebellar tonsils. IV = fourth ventricle; MED OBL = medulla oblongata; PICA = right posterior inferior cerebellar artery; TONS = cerebellar tonsil.

and augmented with fibrin glue. The wound was closed meticulously in layers.

\section{Evaluation of Intraoperative SSEPs}

Somatosensory evoked potentials were recorded continuously throughout the entire operation, from positioning of the patient through wound closure. Stimulation and recording parameters, as well as stimulation and recording sites, are summarized in Table 1.

All electrophysiological data were stored, and completion time points of positioning, craniotomy, fourth ventricle opening, and wound closure were noted for post hoc analyses.

The latencies and the amplitudes of the first negativity of the primary cortical responses (N20) over both hemispheres were evaluated after averaging 100 signals, as illustrated in Fig. 3.

The SSEP signals were observed continuously during the operation by a trained neurophysiologist. Significant changes in N20 amplitudes and N20 latencies were supposed to be communicated to the operative team. A change of 50\% in N20 amplitude from the baseline value, and a change of $10 \%$ in N20 latency from the baseline value were considered as significant for intraoperative use. From

TABLE 1. Stimulation and recording parameters and stimulation and recording sites of SSEPS

\begin{tabular}{lc}
\hline \multicolumn{1}{c}{ Parameter } & Value \\
\hline Stimulation site & Median nerve/wrist \\
\hline Intensity $(\mathrm{mA})$ & 30 \\
\hline Pulse duration $(\mu \mathrm{sec})$ & 200 \\
\hline Frequency $(\mathrm{Hz})$ & 4.7 \\
\hline Averaging $(\mathrm{no}$. of times) & 100 \\
\hline Recording sites/channels & $\mathrm{C} 3^{\prime} / \mathrm{Fpz} ; \mathrm{C} 4^{\prime} / \mathrm{Fpz}$ \\
\hline Low-pass filter $(\mathrm{Hz})$ & 500 \\
\hline High-pass filter $(\mathrm{Hz})$ & 5.0 \\
\hline
\end{tabular}




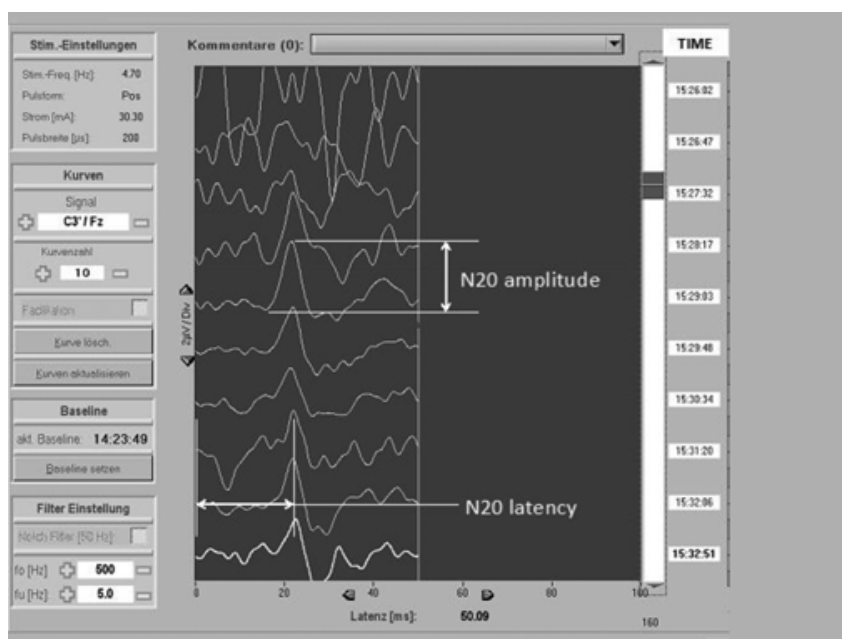

FIG. 3. Screen of an intraoperative neuromonitoring device illustrating continuous SSEP monitoring. N20 latencies and N20 amplitudes (double arrows) were evaluated every 46 seconds, from positioning to wound closure throughout the entire procedure. Signals were stored and completion times of different surgical steps were noted for post hoc analyses.

positioning to completion of dura opening no significant change in SSEP parameters could be observed.

Within a few minutes after opening the fourth ventricle and establishing CSF flow through the foramen of Magendie, a $230 \%$ increase of the N20 amplitude over the baseline value was observed. Simultaneously, an $8 \%$ decrease of the N20 latency from the baseline value was observed. This clear SSEP improvement persisted until the end of the operation, without significant variation. Because of this interesting intraoperative observation, the data were prepared for postoperative analysis as follows.

Over 800 single SSEP values were allocated to specific surgical steps: 1) patient positioning-craniotomy; 2) craniotomy-dura opened; 3 ) dura opened-fourth ventricle opened; and 4) fourth ventricle opened-closure.

The mean N20 latencies and amplitudes of the respective groups are shown in Table 2.

The N20 parameters were tested with a 2-tailed t-test for significance of differences and Table 3 provides a summary of these $p$ values.

A statistically significant improvement in SSEP amplitudes and latencies could not be found after craniotomy or durotomy, as shown in Table 3.

A statistically significant increase of N20 amplitudes
TABLE 3. The $p$ values of the 2-tailed t-test for $\mathrm{N} 20$ amplitudes and latencies at the different stages of CM-I decompression

\begin{tabular}{lccc}
\hline & \multicolumn{3}{c}{$p$ Value } \\
\cline { 2 - 4 } Parameter & $\begin{array}{c}\text { Before vs After } \\
\text { Craniotomy }\end{array}$ & $\begin{array}{c}\text { Before vs After } \\
\text { Durotomy }\end{array}$ & $\begin{array}{c}\text { Before vs After 4th } \\
\text { Ventricle Opening }\end{array}$ \\
\hline N20 amplitudes & 0.239 & 0.455 & $<0.01$ \\
\hline N20 latencies & 0.106 & 0.311 & $<0.01$ \\
\hline
\end{tabular}

and decrease of N20 latency was only seen after opening of the fourth ventricle.

\section{Postoperative Course}

The patient was extubated in the operating room, and she awoke without any cardiorespiratory problems. Neurological examination on the 1st postoperative day revealed significantly improved spinal ataxia and partially improved sensory disturbances compared with the preoperative state. Mild wound pain was treated with acetaminophen for 3 days. The patient was discharged from hospital on the 5th postoperative day.

Postoperative MRI revealed a considerable reduction in syringomyelia (Fig. 1). At the follow-up visits, the patient reported no more episodes of occipital headaches and a further improvement of her sensory disturbances. Spinal ataxia could no longer be detected at the latest follow-up visit 32 months after the operation.

\section{Ethics}

The study has been approved by the local ethics committee. The patient has also given informed consent.

\section{Discussion}

The pathophysiology of CM is poorly understood, its natural history is unpredictable, and there is no robust evidence demonstrating its response to various treatment methods. The surgical techniques applied by various authors for CM-I decompression differ in the extent of the craniotomy and cervical laminectomy, dura opening, arachnoid membrane opening, lysis of arachnoid adhesions, partial tonsillar resection, and the type of duraplasty. ${ }^{6,8}$ ${ }^{10-13}$ Whereas the risk of complications increases with every additional surgical step, the risk of reoperation being needed to treat persistent symptoms decreases in the same way. ${ }^{3}$ Two recent publications, one by Chotai et al. ${ }^{6}$ and the other by Williams et al., ${ }^{13}$ demonstrated similar com-

TABLE 2. The mean amplitudes and latencies of the $\mathrm{N} 20$ at different stages of $\mathrm{CM}-\mathrm{I}$ decompression, from craniotomy through closure of the fourth ventricle

\begin{tabular}{lcccc}
\hline \multirow{2}{*}{ Parameter } & \multicolumn{4}{c}{ Surgical Step } \\
\cline { 2 - 5 } & $\begin{array}{c}\text { Positioning to Craniotomy } \\
+ \text { C-1 Laminectomy }\end{array}$ & $\begin{array}{c}\text { Craniotomy to } \\
\text { Dura Opened }\end{array}$ & $\begin{array}{c}\text { Dura Opened to 4th } \\
\text { Ventricle Opened }\end{array}$ & $\begin{array}{c}\text { 4th Ventricle } \\
\text { Opened to Closure }\end{array}$ \\
\hline Mean amplitude N20 $(\mu \mathrm{V})^{*}$ & $0.8(0.07)$ & $0.8(0.08)$ & $0.8(0.12)$ & $1.9(0.28)$ \\
\hline Mean latency N20 $(\mathrm{msec})^{*}$ & $20.8(0.37)$ & $20.6(0.67)$ & $20.4(0.36)$ & $19.2(0.58)$ \\
\hline Time interval (mins) & 110 & 38 & 57 & 102 \\
\hline No. of averaged signals/hemisphere & 310 & 107 & 160 & 287 \\
\hline
\end{tabular}


plication rates and outcomes following craniotomy, C-1 laminectomy, arachnoid lysis, and duraplasty in series of adult patients. They reported favorable outcomes in $90 \%$ of the patients and an improvement in physical health and function in all patients. The most common complications following this surgical technique are pseudomeningocele formation, CSF leakage, and meningitis in up to $31 \%$, $12 \%$, and $12 \%$ of the cases, respectively. To reduce the risk of pseudomeningocele development, Galarza et al. ${ }^{8}$ performed only very small craniotomies limited to the occipital bone rim in a series of 22 adult patients. To establish CSF flow at the craniocervical junction, they reduced the volume of the cerebellar tonsils through resection, subpial aspiration or repositioning, and duraplasty. A pseudomeningocele could be verified in only $5 \%$ of their patients, whereas most preoperative complaints resolved in all of the patients. In contrast to these surgical approaches, when craniotomy and cervical laminectomy alone are performed and the dura is left intact, these complications virtually do not occur. This less invasive technique is more often applied in pediatric CM patients without associated syringomyelia. According to this principle, Litvack et al ${ }^{10}$ found, in a cohort of pediatric patients treated with duraplasty, that $93.6 \%$ exhibited associated syringomyelia, whereas in a cohort in which dura splitting was performed, only $7.9 \%$ showed associated syringomyelia. They reported similar outcomes in both cohorts in terms of resolution or improvement of presenting complaints in $90 \%$ and $91 \%$ of the patients and a significantly lower complication rate in the dura-splitting cohort. They concluded that $\mathrm{CM}$ with and $\mathrm{CM}$ without syringomyelia represent two different disease entities and therefore should be treated using distinct techniques. Hence, the treatment of $\mathrm{CM}$ associated with syringomyelia includes duraplasty in most cases. Although there is certain agreement among neurosurgeons concerning the appropriate surgical method for some subgroups of CM-I patients, the most beneficial technique cannot be predicted for individual patients.

To shed light on the pathophysiology of this complex condition a few authors have studied the influence of different surgical steps on intraoperative evoked potentials in CM-I posterior fossa decompression. ${ }^{1,5,14}$ Various authors have investigated the consistency of intraoperative evoked potential changes that might aid the surgeon to determine the appropriate extent of decompression required for an individual case. The investigations' main characteristics and findings are shown in Table 4.

Anderson et al. ${ }^{1}$ studied the changes of brainstem auditory evoked potentials (BAEPs) in a small pediatric series in which craniotomy and duraplasty were performed. Conduction times of BAEPs were compared at 4 different time points: before positioning, after positioning, after craniotomy, and after durotomy. The authors found a significant decrease in the BAEP interpeak latencies I-V, I-III, and III-V following craniotomy but no significant additional improvement in conduction times following durotomy. Because the majority of improvement in conduction time through the brainstem occurred before durotomy, just after craniotomy and division of the atlantooccipital membrane, they concluded that their patients might not require durotomy at all. The authors also recorded SSEPs, but they did not analyze their changes at different surgical stages as they did for BAEPs. The authors noted that SSEPs remained stable throughout the operation in all but one case, when a deterioration in SSEP parameters was noticed during positioning.

The same approach was used by Chen et al., ${ }^{5}$ also in a small series of pediatric cases. However, the authors expanded their analyses by evaluating SSEP parameters and found significant conduction changes in SSEPs and BAEPs both following craniotomy and durotomy, in contrast to the report by Anderson et al. ${ }^{1}$ Similar to Anderson et al., Chen et al. performed craniotomy and duraplasty in all cases, replacing the bone flap in most cases.

Zamel et al. ${ }^{14}$ studied the intraoperative changes of BAEPs in a large consecutive series of pediatric and adult patients. Their operative approach differed depending on the presence of syringomyelia. The majority of patients with syringomyelia underwent a craniotomy, C-1 laminectomy, and duraplasty, whereas almost all patients without syringomyelia underwent craniotomy and C-1 laminectomy alone. Their data revealed that for both groups of patients, with or without associated syringomyelia, the predominant improvement in central conduction occurred during the phase when bony decompression was being performed, and no significant additional improvement after duraplasty.

In our case we did not observe a significant improvement in SSEP conduction after craniotomy or duraplasty. Only after opening of the fourth ventricle did SSEP parameters improve significantly. The cause of this extraordinary finding may either lie in the specific pathophysiology of the particular case or, more likely, in methodological differences between our procedure and those of the aforementioned authors. All discussed studies evaluated evoked potential parameters at 4 different times during the operation - that is, before positioning, after positioning, after craniotomy, and wound closure. In contrast, we evaluated continuous SSEP parameters throughout the entire operation, with more than 800 single parameters that were allocated to the different stages of the operation. Because of the high degree of variability of SSEPs in an opera-

TABLE 4. Summary of characteristics and findings of studies reporting evoked potential changes during CM-I decompression

\begin{tabular}{lclll}
\hline \multicolumn{1}{c}{ Authors \& Year } & $\begin{array}{c}\text { No of } \\
\text { Cases }\end{array}$ & Methods Used & \multicolumn{1}{c}{ Parameters Improved } & $\begin{array}{c}\text { Surgical Step Preceding Significant } \\
\text { Electrophysiological Improvement }\end{array}$ \\
\hline Anderson et al., 20032 & 11 & BAEP; SSEP & I-V, I-III, \& III-V interpeak latencies; SSEPs stable & Craniotomy \\
\hline Zamel et al., 2009 & 80 & BAEP & I-V interpeak latencies & Craniotomy \\
\hline Chen et al., 2012 & 13 & BAEP; SSEP & N20 latencies; V latencies & Craniotomy \& durotomy \\
\hline Present case & 1 & SSEP & N20 latencies; N20 amplitudes & Opening 4th ventricle \\
\hline
\end{tabular}


tive environment, the electrophysiological condition of the central nervous system could possibly be reflected more accurately by assessing continuous recordings. Furthermore, we hypothesize that the improvement in conduction was due to a restoration of CSF flow at the craniocervical junction and to relieved pressure on the spinal cord caused by the syringomyelia at the stage of surgery when the fourth ventricle was opened. This decompression of the ascending pathways of the spinal cord can be monitored using SSEPs, whereas BAEPs indicate decompression at the level of the brainstem.

Because the reported procedures were not tailored to individual potential changes, the findings are somewhat restricted in their prognostic value and cannot be safely extrapolated to show that the observed improvements in intraoperative evoked potentials are a good indicator of postoperative clinical improvement.

Our data support that prospective studies are warranted to clarify not only the precise role of electrophysiological monitoring, but also which degree of decompression each patient will require.

\section{Conclusions}

Improvement of SSEP conduction during CM-I decompression may not always occur after bony decompression or duraplasty but may only occur following opening of the fourth ventricle and establishing CSF flow at the level of the craniocervical junction. This indicates that there might be a subset of CM-I patients who require subarachnoid dissection at the level of the craniocervical junction to benefit clinically. Additional studies are needed to be able to tailor the degree of decompression to each CM-I patient based on intraoperative electrophysiological data.

\section{References}

1. Anderson RC, Dowling KC, Feldstein NA, Emerson RG: Chiari I malformation: potential role for intraoperative electrophysiologic monitoring. J Clin Neurophysiol 20:65-72, 2003

2. Anderson RC, Emerson RG, Dowling KC, Feldstein NA: Improvement in brainstem auditory evoked potentials after suboccipital decompression in patients with Chiari I malformations. J Neurosurg 98:459-464, 2003

3. Baisden J: Controversies in Chiari I malformations. Surg Neurol Int 3 (Suppl 3):S232-S237, 2012

4. Caldarelli M, Novegno F, Vassimi L, Romani R, Tamburrini G, Di Rocco C: The role of limited posterior fossa craniectomy in the surgical treatment of Chiari malformation Type I: experience with a pediatric series. J Neurosurg 106 (3 Suppl):187-195, 2007

5. Chen JA, Coutin-Churchman PE, Nuwer MR, Lazareff JA: Suboccipital craniotomy for Chiari I results in evoked potential conduction changes. Surg Neurol Int 3:165, 2012
6. Chotai S, Kshettry VR, Lamki T, Ammirati M: Surgical outcomes using wide suboccipital decompression for adult Chiari I malformation with and without syringomyelia. Clin Neurol Neurosurg 120:129-135, 2014

7. Durham SR, Fjeld-Olenec K: Comparison of posterior fossa decompression with and without duraplasty for the surgical treatment of Chiari malformation Type I in pediatric patients: a meta-analysis. J Neurosurg Pediatr 2:42-49, 2008

8. Galarza M, Gazzeri R, Alfieri A, Martínez-Lage JF: “Triple R" tonsillar technique for the management of adult Chiari I malformation: surgical note. Acta Neurochir (Wien) 155:1195-1201, 2013

9. Isu T, Sasaki H, Takamura H, Kobayashi N: Foramen magnum decompression with removal of the outer layer of the dura as treatment for syringomyelia occurring with Chiari I malformation. Neurosurgery 33:845-850, 1993

10. Litvack ZN, Lindsay RA, Selden NR: Dura splitting decompression for Chiari I malformation in pediatric patients: clinical outcomes, healthcare costs, and resource utilization. Neurosurgery 72:922-929, 2013

11. Ma J, You C, Chen H, Huang S, Ieong C: Cerebellar tonsillectomy with suboccipital decompression and duraplasty by small incision for Chiari I malformation (with syringomyelia): long term follow-up of 76 surgically treated cases. Turk Neurosurg 22:274-279, 2012

12. Parker SL, Godil SS, Zuckerman SL, Mendenhall SK, Tulipan NB, McGirt MJ: Effect of symptomatic pseudomeningocele on improvement in pain, disability, and quality of life following suboccipital decompression for adult Chiari malformation Type I. J Neurosurg 119:1159-1165, 2013

13. Williams LE, Vannemreddy PS, Watson KS, Slavin KV: The need in dural graft suturing in Chiari I malformation decompression: a prospective, single-blind, randomized trial comparing sutured and sutureless duraplasty materials. Surg Neurol Int 4:26, 2013

14. Zamel K, Galloway G, Kosnik EJ, Raslan M, Adeli A: Intraoperative neurophysiologic monitoring in 80 patients with Chiari I malformation: role of duraplasty. J Clin Neurophysiol 26:70-75, 2009

\section{Author Contributions}

Conception and design: Grossauer. Acquisition of data: Koeck. Drafting the article: Grossauer. Critically revising the article: Vince. Reviewed submitted version of manuscript: Vince. Statistical analysis: Koeck.

\section{Supplemental Information}

\section{Previous Presentation}

Portions of this work were presented in abstract form at the Neurophysiological Section Meeting of the German Society of Neurological Surgeons, Wuerzburg, Germany, January 18, 2014.

\section{Correspondence}

Stefan Grossauer, Department of Neurosurgery, General Hospital Klagenfurt, Feschnigstrasse 11, A-9020 Klagenfurt, Austria. email: st.grossauer@gmail.com. 